\title{
Adaptive cognitive radio energy-harvesting scheme using sequential game approach
}

\author{
Sungwook Kim
}

\begin{abstract}
Radio frequency (RF) energy transfer and harvesting techniques have recently become alternative methods to overcome the barriers that prevent the real-world wireless device deployment. For the next-generation wireless networks, they can be key techniques. In this study, we develop a novel energy-harvesting scheme for the cognitive radio (CR) network system. Using the sequential game model, data transmission and energy harvesting in each device are dynamically scheduled. Our approach can capture the wireless channel state while considering multiple device interactions. In a distributed manner, individual devices adaptively adjust their decisions based on the current system information while maximizing their payoffs. When the channel selection collision occurs, our sequential bargaining process coordinates this problem to optimize a social fairness. Finally, we have conducted extensive simulations. The results demonstrate that the proposed scheme achieves an excellent performance for the energy efficiency and spectrum utilization. The main contribution of our work lies in the fact that we shed some new light on the trade-off between individual wireless devices and CR network system.
\end{abstract}

Keywords: Energy harvesting, Radio frequency, Cognitive radio network, Game theory, Sequential bargaining solution

\section{Introduction}

Wireless communication network is becoming more and more important and has recently attracted a lot of research interest. Compared with wireline communication, wireless communication has a lower cost, which is easier to be deployed. With the development of Internet of Things (IoT) and embedded technology, wireless communication will be applied in more comprehensive scopes. Sometimes, wireless communications work in the license-free band. As a result, it may suffer from heavy interference caused by other networks sharing the same spectrum. In addition, wireless devices perform complex task with portable batteries. However, batteries present several disadvantages like the need to replace and recharge periodically. As the number of electronic devices continues to increase, the continual reliance on batteries can be both cumbersome and costly [1-4].

Recently, radio frequency (RF) energy harvesting has been a fast growing topic. The RF energy harvesting is developed as the wireless energy transmission technique

Correspondence: swkim01@sogang.ac.kr

Department of Computer Science, Sogang University, 35 Baekbeom-ro (Sinsu-dong), Mapo-gu, Seoul 121-742, South Korea for harvesting and recycling the ambient RF energy that is widely broadcasted by many wireless systems such as mobile communication systems, Wi-Fi base stations, wireless routers, wireless sensor networks, and wireless portable devices [5]. Therefore, this technique becomes a promising solution to power energy-constrained wireless networks while allowing the wireless devices to harvest energy from RF signals. In RF energy harvesting, radio signals with frequency range from $300 \mathrm{GHz}$ to as low as $3 \mathrm{kHz}$ are used as a medium to carry energy in a form of electromagnetic radiation. With the increasingly demand of RF energy harvesting/charging, commercialized products, such as Powercaster and Cota system, have been introduced in the market [6].

In wireless communication, cognitive radio (CR) technology has evolved to strike a balance between the underutilized primary user (PU)'s spectrum band and the scarcity of spectrum band due to increased wireless applications. To improve the spectrum utilization efficiency, CR networks make secondary user (SU) exploit the underutilized PU's band. Nowadays, the CR technology has employed the RF energy-harvesting capability that enables SUs to opportunistically not only transmit 
data on an idle channel, but also harvest RF energy from PUs' transmission on a busy channel [7-9].

Powering a cognitive radio network with RF energy (CRN-RF) system can provide a spectrum-energy efficient solution for wireless communications. In the CRN-RF system, the dual use of RF signals for delivering energy as well as for transporting information has been advocated. Therefore, wireless devices must not only identify spectrum holes for opportunistic data transmission but also search for occupied spectrum band to harvest RF energy. Such RF signals could be from nearby non-battery powered base stations or access points. The RF signal can be converted into DC electricity, and it can be stored in a battery for the information processing and data transmission. This approach can offer a low-cost option for sustainable operations of wireless systems without hardware modification on the transmitter side. However, due to the specific nature of CRN-RF, traditional CRN protocols may not be directly applied. In addition, the amount of transmitted information and transferred energy cannot be generally maximized at the same time. Therefore, the main challenge in CRN-RF system is to strike a well-balanced trade-off between data transmission and RF energy harvesting [6, 7].

Usually, all network agents in the CRN-RF system are assumed to work together in a coordinated manner. However, in practice, network devices are always selfish individuals that will not contribute their work without getting paid. This situation can be seen a game theory paradigm. Game theory is a decision-making process between independent decision-making players as they attempt to reach a joint decision that is acceptable to all participants. In the game theory, a solution concept is a rule that defines what it means for a decision vector to be acceptable to all players in the light of the conflictcooperation environment. Nowadays, many applications of the game theory are related to a wider range of wireless communications and network managements [10].

Motivated by the above discussion, we propose a new energy-harvesting scheme for CRN-RF systems. To develop a practical control mechanism, we adopt a game theory model to design the interactive relationship among the system agents. Under the real-world CRN-RF environments, the system agents are mutually dependent on each other to maximize their payoffs. In the proposed scheme, system agents dynamically adjust their control decisions while responding individually to the current system situations in order to maximize their payoffs. This interactive procedure imitating the sequential game process is practical and suitable for real-world CRN-RF implementation. In addition, we consider the social fairness issue among network devices. Therefore, the channel spectrum is shared adaptively according to the sequential bargaining mechanism. In realistic point of view, our approach can be implemented with reasonable complexity.

The major contributions of our proposed scheme are (i) the adjustable dynamics considering the current CRN-RF system environments, (ii) the ability to strike an appropriate trade-off in harvesting energy and transferring data, (iii) practical approach to effectively reach a desirable solution, (iv) dynamic interactive process in a distributed fashion, and (v) the ability to maximize the total system performance by incorporating the social fairness. In the proposed scheme, we can effectively address the energy-harvesting problem by using the practical sequential game model while better capturing the reality of wireless communications; it has never been studied in the previous literatures.

\subsection{Related work}

Over the years, extensive research on efficient data transmission and RF energy harvesting has been carried out. Niyato et al. [11] presented an overview of the different energy-harvesting technologies and the energy-saving mechanisms for wireless sensor networks. By using the energy-harvesting technology, the issues on energy efficiency for sensor networks were intensively discussed. Finally, they showed an optimal energy management policy for a solar-powered sensor node that used a sleep and wakeup strategy for energy conservation [11].

In [12], authors investigated energy-effient uplink power control and subchannel allocation algorithms for two-tier femtocell networks. Based on the supermodular game model, they addressed the power control and subchannel allocation problem while maximizing energy effiency of femtocell users. To reduce costs and complexity, the resource allocation problem was decomposed into two subproblems, that is, a distributed subchannel allocation scheme and a distributed power control scheme [12].

Zhang et al. [13] investigated the joint uplink subchannel and power allocation problem in cognitive small cells. By using the cooperative Nash bargaining theory, their approach mitigated the cross-tier interference, minimized the outage probability, and ensured the fairness in terms of minimum rate requirement. Based on the Lagrangian dual decomposition by introducing time-sharing variables and the Lambert-W function, the near optimal cooperative bargaining resource allocation strategy was derived. Finally, the existence, uniqueness, and fairness of their solution were proved [13].

In [14], authors proposed a distributed power control scheme for the uplink transmission of spectrum-sharing femtocell networks. According to a fictitious game model, each network user announced a price that reflected its sensitivity to the current interference level and adjusted its power to maximize its utility. The convergence to a 
unique optimal equilibrium was proved. Furthermore, simple macrocell link protection and power optimization schemes were developed for the effective resource allocation in spectrum-sharing two-tier networks [14].

Authors in [15] investigated the uplink resource allocation problem of femtocells in co-channel deployment with macrocells. They modeled the uplink power and subchannel allocation in femtocells as a non-cooperative game. Based on this game model, they devised a semi-distributed algorithm for each femtocell to first assign subchannels to femto users and then allocated power to subchannels. Finally, they showed that their interference-aware femtocell uplink resource allocation algorithm was able to provide improved capacities for not only femtocells but also the macrocell in the two-tier network [15]. All the earlier work in [11-15] addressed the state-of-the-art research issue. However, the work in [11] provided an overview about the energy-harvesting technology, and others [12-15] strongly focused on the power control problem while considering optimal solutions. Due to the model complexity, optimal solution approaches are impractical to be implemented for realistic system operations.

The optimized node classification and channel pairing (ONCCP) scheme [16] merged the cognitive radio and $\mathrm{RF}$ energy-harvesting technologies together to achieve network-wide spectral and energy efficiency. A novel two-level node-classification algorithm was introduced to select the best wireless nodes for reporting process. At first level, the nodes were classified as harvesting or transmitting nodes based on their residual energy. In the second level, only those nodes could perform reporting, which acquired better quality channels that could transmit reporting-packet within the designated data slots. By employing two-level classification, the ONCCP scheme ensured successful reporting probability and achieved energybalancing [16].

The opportunistic channel access and energy-harvesting (OCAEH) scheme [8] considered a network where the secondary user could perform channel access to transmit a packet or to harvest RF energy when the selected channel was idle or occupied by the primary user, respectively. And then, the optimization formulation was presented based on Markov decision process to obtain the channel access policy. This formulation did not need the secondary user to know the current channel status. However, the optimization problem required various model parameters to obtain the policy. To obviate such a requirement, the OCAEH scheme applied an online learning algorithm that could observe the environment and adapted the channel access action accordingly without any a prior knowledge about the model parameters [8]. The ONCCP and OCAEH schemes in $[8,16]$ have attracted a lot of attention and introduced unique challenges to efficiently handle the CRN-RF system. In this study, we compare the performance of our proposed scheme with the existing schemes in $[8,16]$ through extensive simulation. The analysis is given in Section III.

The rest of this paper is organized as follows. In Section II, we familiarize the reader with the basics of energyharvesting game model and explain in detail the developed CRN-RF scheme based on the sequential game procedure. We present experimental results in Section III and compare the performance to other existing schemes $[8,16]$. Finally, we give our conclusion and future work in Section IV.

\section{Cognitive radio network energy-harvesting algorithm}

In this section, we present an energy-harvesting game model for the CRN-RF system. Our game model employs a repeated interactive procedure while considering current system conditions. And then, we explain in detail about the proposed algorithm in the nine-step procedures.

\subsection{Energy-harvesting game model}

To model an energy-harvesting game for the CRN-RF system, it is assumed that a cognitive radio network with multiple PUs and SUs; PUs are licensed and non-batterypowered network agents, and SUs are unlicensed network devices operating by batteries. For each PU, a nonoverlapping spectrum channel is allocated individually, and a channel can be free or occupied by the PU for data transmission. SUs have the RF energy-harvesting capability and perform the channel access by selecting one of them. If the selected channel is busy, the SU can harvest RF energy; the harvested energy is stored in the SU's battery. Otherwise, the SU can transmit his data packets [8].

Simply, we consider a CRN-RF system composed one cognitive base station (CBS), $m$ PUs, and $n$ SUs $(m<n)$. Based on the TDMA mechanism, the licensed spectrum channel consists of sequential frames. In other word, each spectrum channel is divided as multiple frames [17]. Frames over time are used for data transmission or RF energy transferring. Under dynamic network environments, the PU can use the whole frame for his own data transmission or vacates his frames. When a frame is actively used for the PU's data transmission, the RF energy can be transferred to the SUs through the RF energy harvesting process. If the frame is vacated, this vacated frame can be used by a SU. The illustrative structure of channel frames is shown in the Fig. 1.

In this study, we define a new game model, called energy-harvesting game $(\mathbb{G})$, based on the sequential game approach. This new game model is originally designed to harvest the energy for the CRN-RF system. To effectively model strategic CRN-RF situations, we assume that unlicensed network devices, i.e., SUs, are game players. During the interactive sequential game process, players choose their strategy based on the reciprocal relationship. 


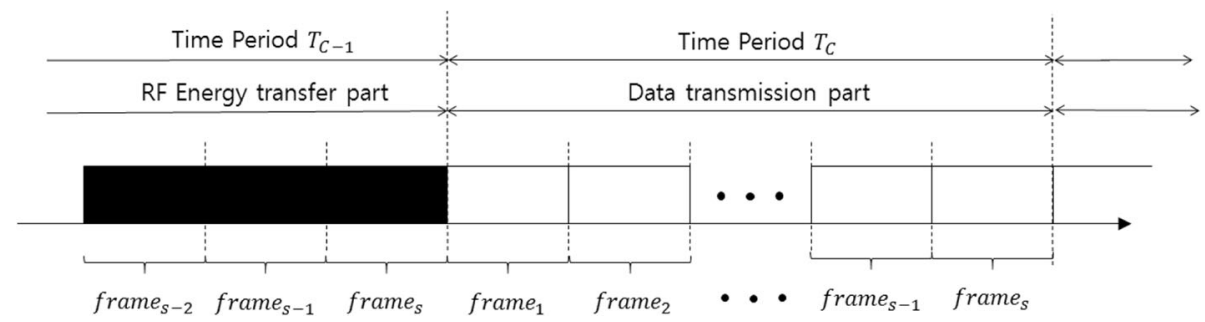

Fig. 1 Spectrum channel structure with multiple frames

From the view of individual SUs, the main challenge is to effectively transmit his data or to harvest RF energy. Therefore, the proposed energy-harvesting game $\mathbb{G}$ is designed as a symmetric game with the same strategy set for game players.

Definition 1. The energy-harvesting game $(\mathbb{G})$ constitutes three game tuple $\left(\mathbb{G}=\boldsymbol{N}, \quad \boldsymbol{S}_{i, 0 \leq i \leq n}, \quad \boldsymbol{U}_{i, 0 \leq i \leq n}\right)$, where

(i) $N$ is a set of game players; $i \in N=\{1, \ldots, n\}$ is an unlicensed network device in the CRN-RF system

(ii) $S_{i}=\left\{s_{i}^{1}, \ldots s_{i}^{k} \ldots s_{i}^{m}\right\}$ is a non-empty finite strategy set of the player $i \in N ; s_{i}^{k}$ means that the $k^{\text {th }}$ PU channel is selected by the player $i$ for the CRN-RF service

(iii) $U_{i}$ is the utility function to represent the payoff of player $i \in N$. $U_{i}$ is decided according to the set of all players' strategies: $\left(s_{1}^{(\cdot)} \times \ldots s_{i}^{(\cdot)} \ldots \times s_{n}^{(\cdot)}\right) \rightarrow U_{i}$.

In our game model $\mathbb{G}$, each PUs arbitrarily use their channels to transmit their data over time. Therefore, SUs can temporally access the PUs' channels in an opportunistic manner. As the data sender or RF energy harvester, the major goal of SUs is to jointly optimize the data transmission and energy harvesting. If SUs want to transmit their data, they try to find the vacant PU channels. If multiple SUs access a specific vacant channel at the same time, the channel frames are adaptively distributed for each SU to maximize the total CRN-RF system performance. If the PU comes back to use his designated channel, SUs should release the momentaryusing channel and try to find other idle channels $[6,8]$. If SUs want to harvest the energy, they try to find the active data transferring channels of PUs.

In this study, we assume a practical energy consumption and harvesting model for wireless networks. For a packet transmitted from a transmitter device to a receiver device, the energy $\left(E_{t}\right)$ consumed at the packet transmitter is defined as [18];

$$
E_{t}\left(\mathfrak{F}_{n}, \mathcal{N}_{p}\right)=\left(\left(e_{e} \times \mathfrak{F}_{n}\right)+\left(p l_{t} \times \mathscr{L}\right)\right) \times \mathcal{N}_{p}
$$

where $e_{e}$ is the energy consumed by the device electronics per bit, and $\mathfrak{F}_{n}$ is the bit number of a packet. $p l_{t}$ is a transmission power level, and $\mathscr{L}$ is the bit period. $\mathcal{N}_{p}$ is the total number of packets. Let $t_{c}$ be the current time, and $t_{c+1}$ be the beginning time of next time period. If the device $i$ transmits $\mathcal{N}_{p}^{i}$ packets during $\left[t_{c}, t_{c+1}\right]$ time period, the device $i$ 's expected residual energy at the time $t_{c+1}\left(\Phi_{i}\left(t_{c+1}\right)\right)$ can be computed as follows:

$$
\Phi_{i}\left(t_{c+1}\right)=\Re_{i}\left(t_{c}\right)-E_{t}\left(\mathfrak{\Im}_{n}, \mathcal{N}_{p}^{i}\right)
$$

where $\mathfrak{R}_{i}\left(t_{c}\right)$ is the device $i$ 's residual energy level at time $t_{c}$. In contrast to the energy consumption, the energy harvesting is the process by which energy is derived from external sources and stored in wireless devices. If a PU actively transmits his own data during the time period $\left[t_{c}, t_{c+1}\right]$, the RF energy-harvesting rate $\left(\mathscr{E}^{H}\left(t_{c}, t_{c+1}, d\right)\right)$ in SUs is given as follows $[16,18]$;

$$
\mathscr{E}^{\mathbb{E} H}\left(t_{c}, t_{c+1}, d\right)=\int_{t=t_{c}}^{t=t_{c+1}}\left(\Gamma \times p_{t} \times\left(\frac{G_{t} \times G_{r}}{(4 \pi)^{2} \times d^{\alpha}}\right)\right) d t
$$

where $\Gamma$ is a factor of energy-harvesting efficiency, and $p_{t}$ is the signal transmission power of the PU with RF resource. $G_{t}$ and $G_{r}$ are the gains of the energy transmitter and receiver, respectively. $d$ is the distance between the RF source and the energy-receiving device, and $\alpha$ is the path-loss exponent.

Fundamental problem in game theory is determining how players reach their decisions. Usually, players selfishly select a strategy that maximizes their own payoff through the utility function. To design the utility function $\left(U_{i}\right)$ of player $i$, we should define the received benefit and the incurred cost during the CRN-RF services. Let $\beta_{i, k}\left(p l_{t}^{i}\right)$ be the data transmission amount that the player $i$ can achieve over the $k^{\text {th }}$ frame in a channel with the power level $p l_{t}^{i}$. Using Shannon formula, the player $i$ 's time-varying data transmission amount can be computed as follows:

$$
\beta_{i, k}\left(p l_{t}^{i}\right)=W_{k} \times\left(\log _{2}\left(1+\gamma_{i, k}\left(p l_{t}^{i}\right)\right)\right)
$$

where, $W_{k}$ is the bandwidth of $k^{\text {th }}$ frame and $\gamma_{i, k}\left(p l_{t}^{i}\right)$ is the signal-to-interference-and-noise ratio (SINR) over 
the $k^{\text {th }}$ frame [16]. The cost is defined as the instantaneous expense function of consumed energy. Finally, the player $i$ 's utility function $\left(U_{i}(\cdot)\right)$ during $\left[t_{c}, t_{c+1}\right]$ is defined as follows;

$$
\begin{aligned}
& U_{i}\left(p l_{t}^{i}, t_{c}, t_{c+1}\right) \\
& =\left\{\begin{array}{r}
\sum_{k \in \mathrm{S}_{i}, t_{c+1}}\left\{\left(\beta_{i, k}\left(p l_{t}^{i}\right)\right)-\xi \times\left(E_{t}^{i}\left(\widetilde{\mho}_{n}, \mathcal{N}_{p^{i, k}}\right)\right)^{q}\right\}, \\
\text { if data transmission strategy is selected } \\
\mathscr{E}^{\mathscr{C}}\left(t_{c}, t_{c+1}, d\right), \text { if energy harvesting strategy is selected }
\end{array}\right.
\end{aligned}
$$

where $\mathbb{S}_{i}^{t_{c}, t_{c+1}}$ is the set of frames, which are allocated for the player $i$ during $\left[t_{c}, t_{c+1}\right] \cdot p l_{t}^{i}$ and $E_{t}^{i}(\cdot)$ are the power level and the $E_{t}$ value of the player $i$, respectively. $\xi$ and $q$ are cost parameters. $\mathcal{N}_{p^{i, k}}$ are the total number of packets in the $k^{\text {th }}$ frame of the player $i$.

\subsection{Cognitive radio sensing mechanism}

Usually, allocated spectrum channels are largely unused in any time and location; these are referred to as spectrum holes. The increased spectrum utilization in CR networks is achieved through opportunistically sharing the spectrum holes between licensed and unlicensed wireless devices [10]. To detect spectrum holes, unlicensed wireless devices are sensing constantly the allocated spectrum channel. Based on the received signal from the detection, we can define two hypotheses for the case of the licensed device is present $\left(H_{1}\right)$ or absent $\left(H_{0}\right)$. If there are $K$ detection samplings, the $k^{\text {th }}$ received signal for the channel $l$ $\left(r_{l}^{k}\right.$, i.e., $\left.1 \leq k \leq K\right)$ can be written as

$$
r_{l}^{k}=\left\{\begin{array}{cl}
\left(h^{k} \times s(k, l)\right)+w(k, l), & \text { if } H_{1} \\
w(k, l), & \text { if } H_{0}
\end{array}\right.
$$

where $h^{k}$ is the channel gain from the licensed wireless device to the unlicensed wireless device; it is assumed to be slow flat fading. $(k, l)$ is the channel $l$ 's signal of the licensed wireless device, and $(w(k, 1))$ is the channel $l$ 's additive white Gaussian noise (AWGN) with mean zero and variance $\sigma_{w}^{2}$ [19]. In this study, $s(k, l)$ and $w(k, 1)$ are assumed to be mutually independent. In CR systems, each unlicensed wireless device senses the channel and sends the sensing information $r$ to the CBS. Based on the $r_{l}^{k}$ information, the CBS defines the channel $l$ 's test statistics $(\mathfrak{N}(l))$ as follows:

$$
\mathfrak{N}(l)=\frac{1}{K} \times\left(\sum_{k=1}^{K}\left(r_{l}^{k}\right)^{2}\right)
$$

where $K$ is the total number of collected samples [19]. The CBS maintains a look-up vector $(\mathbb{V})$ for each channels. In the proposed scheme, $\mathbb{V}$ represents the $\mathfrak{N}$ (.) outcomes of the last $x$ time rounds $\left[t_{c-x}, t_{c-1}\right]$ while emphasizing the most recent information. By using an appropriate time-oriented fusion rule, the values in $\mathbb{V}$ are estimated in the timed weighted manner. At time $t_{c}$, the $\mathbb{V}$ value for the channel $l\left(\mathbb{V}^{t_{c}}[l]\right)$ is given by;

$$
\mathbb{V}^{t_{c}}[l]=\sum_{f=t_{c-x}}^{t_{c-1}}\left((1-\mu)^{t_{c}-f} \times \mathfrak{P}^{f}[l]\right)
$$

where $\mu$ is a control factor, which can control the weight for each timed strategies, and $\mathfrak{N}^{f}[l]$ is the $\mathfrak{N}[l]$ at the time $f$. Based on the $\mathbb{V}[\cdot]$ information, game players individually decide which PU's channel is selected to maximize their payoffs. To adaptively make these decisions, each player has a data queue $(\mathbb{Q})$ and battery $(\mathbb{E})$. $\mathbb{Q}$ is used to store the generated data for transmission, and $\mathbb{E}$ is a battery to store RF energy harvested from radio signal [8]. $\mathfrak{I}$ and $\mathfrak{P}$ $\left(0 \leq \mathfrak{I} \leq \mathscr{M}^{Q}\right.$ and $\left.0 \leq \mathfrak{P} \leq \mathscr{M}^{\mathbb{E}}\right) \quad$ represent the packet amount in the $\mathbb{Q}$, and energy level of the $\mathbb{E}$, respectively; $\mathscr{M}^{\mathbb{R}}$ is the maximum size of $\mathbb{Q}$ and $\mathscr{M}^{\mathbb{E}}$ is the maximum capacity of $\mathbb{E}$.

Based on the $\mathbb{V}[\cdot], \mathfrak{I}$ and $\mathfrak{P}$ values, each player selects his strategy whether to transmit data or to harvest energy. To formulate the player $i$ 's channel selection problem at the time $t_{c}$, let $\eta_{j}^{i}\left(t_{c}\right)$ is the player $i$ 's propensity for the $j$ 's channel selection. Based on the sophisticated combination of the remaining energy and data arriving rate, $\eta_{j}^{i}\left(t_{c}\right)$ is dynamically estimated for each individual player.

$$
\begin{aligned}
\eta_{j}^{i}\left(t_{c}\right)= & {\left[\frac{\mathfrak{I}}{\mathscr{M}^{\mathbb{Q}}} \times\left((\mathfrak{M}-\mathbb{V}[j]) \times\left(\sum_{l=1}^{M}(\mathfrak{M}-\mathbb{V}[l])\right)^{-1}\right)\right] } \\
+ & {\left[\left(\frac{\mathscr{M}^{\mathbb{E}}-\mathfrak{P}}{\mathscr{M}^{\mathbb{E}}}\right) \times\left(\mathbb{V}[j] \times\left(\sum_{l=1}{ }^{m} \mathbb{V}[l]\right)^{-1}\right)\right], \quad(9) } \\
& \text { s.t., } \mathfrak{M}=\max _{1 \leq l \leq M} \mathbb{V}[l]
\end{aligned}
$$

where $m$ is the total number of PU channels. According to (9), the channel $j$ 's selection probability $\left(\mathcal{P}_{j}^{i}\right)$ from the player $i$ at time $t_{c}$ can be captured as follows:

$$
\mathcal{P}_{j}^{i}\left(t_{c}\right)=\eta_{j}^{i}\left(t_{c}\right) /\left(\sum_{l=1}^{m} \eta_{l}^{i}\left(t_{c}\right)\right)
$$

For the data transmission, individual players try to select a vacant PU channel. If multiple players select the same vacant PU channel, we should decide how to share this licensed spectrum channel to multiple SU. To solve this problem, we adopt the sequential bargaining solution (SBS), which can combine the bargaining problem with social fairness. During continuative time period, SBS process can occur sequentially to capture the strategic interaction among players [20]. Based on the player's I 
value, each player tradeoffs to yield the maximum social benefit. Let $Q=\{1 \ldots i \ldots r \ldots\} \subseteq N$ be the set of players selecting the same channel, the SBS at time $t_{c}\left(\operatorname{SBS}\left(t_{c}\right)\right)$ is $\partial\left(t_{c}\right)=\left\{\partial_{1}^{t_{c}} \ldots \partial_{i}^{t_{c}} \ldots \partial_{r}^{t_{c}}\right\}$ where $\partial_{i}^{t_{c}}$ represents the amount of frames assigned to the player $i$ during $\left[t_{c}, t_{c+1}\right]$. The $\operatorname{SBS}\left(t_{c}\right)$ is obtained through the following maximization problem;

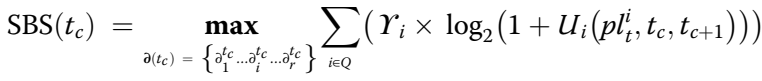

$$
\begin{aligned}
& \text { s.t., } \sum_{i \in Q} \Upsilon_{i}=1 \text { and } \Upsilon_{i}=\mathfrak{I}_{i} / \sum_{i \in Q} \mathfrak{I}_{i}
\end{aligned}
$$

where $\Upsilon_{j}$ is the bargaining power of the player $j$; the bargaining power is the relative priority of the player in the assignment of frames for the bargaining solution. In the proposed scheme, the bargaining powers are decided according to the players' $\mathfrak{I}$ values. If a player has a relatively higher data amount in his $\mathbb{Q}$, he has a higher bargaining power. Therefore, over the time period, the players' $\mathfrak{I}$ values can be balanced while ensuring social fairness among players.

\subsection{The main game procedure in our proposed algorithm} Opportunistic RF energy harvesting is a promising technique to sustain the operation of unlicensed network devices in CRN with RF energy systems. In this study, we focus on the problem of channel selection problem for dynamic spectrum access in a multi-channel CRN-RF system. By considering the trade-off between data transmission and RF energy harvesting, each unlicensed device selects a particular channel to transmit data or harvest RF energy. When the data collusion occurs in a specific channel, available spectrum frames are adaptively distributed through the sequential bargaining process.

Usually, optimal solutions have exponential time complexity. Therefore, they are impractical in real-time process [21, 22]. In the proposed scheme, we do not focus on trying to get an optimal solution based on the traditional approach. Usually, the traditional optimal algorithms have exponential time complexity. However, based on the interactive repeated process, our solution concept only needs polynomial time complexity to capture an effective solution. The proposed algorithm is described by the following major steps and a flow diagram in Fig. 2.

Step 1: At the initial time $t_{c}=1$, the channel selection probability $\mathcal{P}\left(t_{c}\right)$ in each player is equally distributed. This starting guess guarantees that each licensed spectrum channel is selected randomly at the beginning of the game. Step 2: Control parameters $n, m, e_{e}, \mathscr{L}, \Gamma, G_{t}, G_{r}$, $W, \xi, q$ and $\mu$ are given from the simulation scenario (refer to the Table 1).
Step 3: During our iterative dynamic game process, each unlicensed network devices are assumed game players. They sense the spectrum channels and send the $r$ information to the CBS.

Step 4: According to Eqs. (6) and (7), the CBS calculates each channel's test statistics $(\mathfrak{N}(\cdot))$ using the time-oriented fusion rule and maintains a look-up vector $(\mathbb{V})$ for each channels.

Step 5: In an entirely distributed manner, each game player keeps its own control parameters $\mathfrak{I}$ and $\mathfrak{P}$, which are constantly updated. Based on the $\mathbb{V}[\cdot], \mathfrak{I}$ and $\mathfrak{P}$ values, the propensity of each game player $(\eta(\cdot))$ for each channel is estimated using Eq. (9).

Step 6: According to the $\eta(\cdot)$ values, the channel selection probability $(\mathcal{P}(\cdot))$ is dynamically adjusted at each time period. Based on the $\mathcal{P}(\cdot)$, players select the most adaptable spectrum channel to maximize their own payoff.

Step 7: During the step-by-step iteration, players individually adjust their strategies by using the dynamics of repeated game process.

Step 8: When the data collusion occurs in a specific channel, available spectrum frames are dynamically distributed based on the SBS process. Using formula (11), the bargaining power of each player is adaptively adjusted by considering the social fairness.

Step 9: Under the real-world RF-powered CRN environments, the game players are mutually dependent on each other to maximize their profits, and they constantly are self-monitoring the current system conditions; proceeds to Step 3 for the next game iteration.

\section{Performance evaluation}

In this section, we compare the performance of our scheme with other existing schemes $[8,16]$ and can confirm the performance superiority of the proposed approach by using a simulation model. To facilitate the development and implementation of our simulator, Table 1 lists the system control parameters.

Our simulation results are achieved using MATLAB, which is widely used in academic and research institutions as well as industrial enterprises. To ensure the model is sufficiently generic to be valid in a real-world CRN-RF system scenario, the assumptions implemented in our simulation model were as follows.

- The simulated system is assumed as a TDMA system with one macro cell.

- The simulated system consists of one CBS, $m$ number of primary users and $n$ number of secondary users for the CRN-RF system.

- The PUs and SUs are randomly distributed over the $500 \times 500 \mathrm{~m}^{2}$ cell area. 


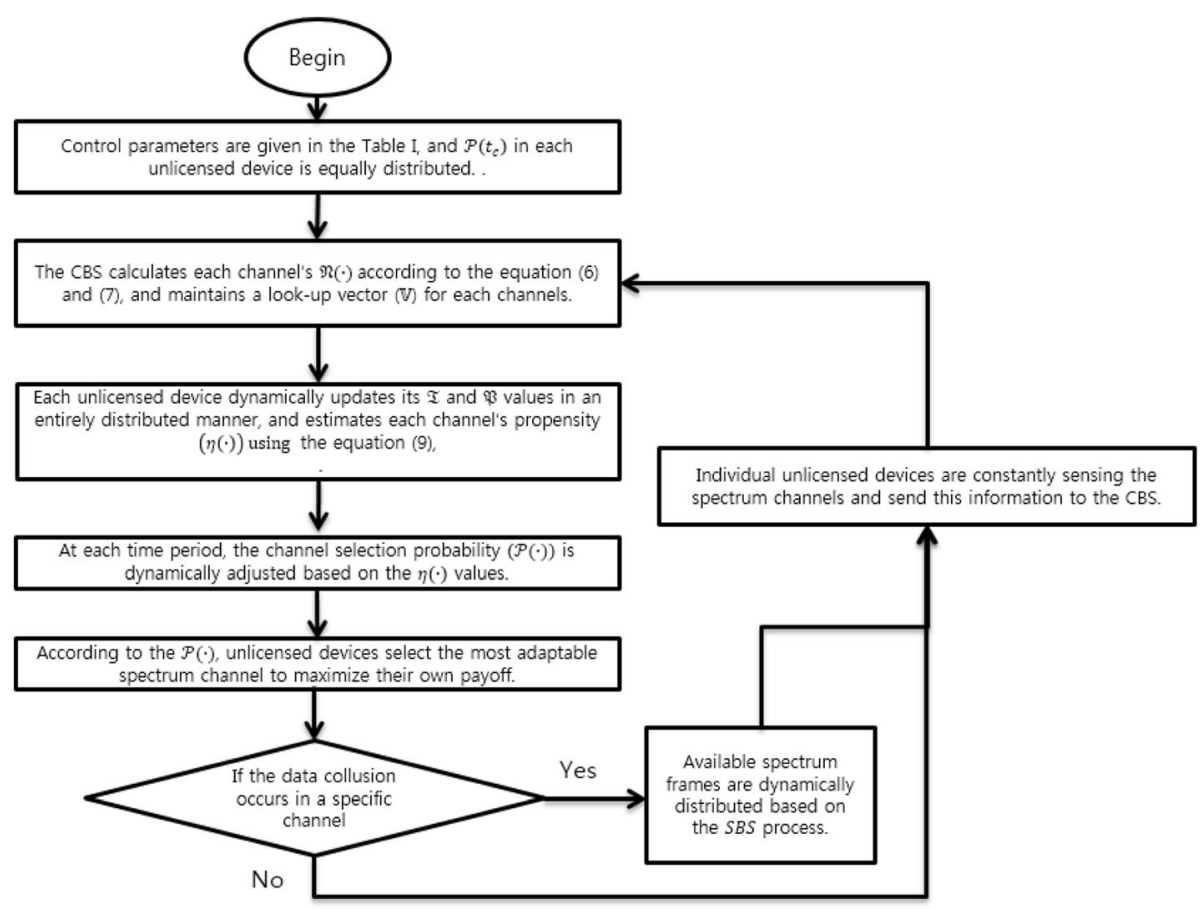

Fig. 2 Flow diagram for the proposed algorithm

Table 1 System parameters used in the simulation experiments

\begin{tabular}{|c|c|c|}
\hline Parameter & Value & Description \\
\hline$n$ & 30 & $\begin{array}{l}\text { The number of secondary users } \\
\text { (unlicensed network devices) }\end{array}$ \\
\hline$m$ & 10 & $\begin{array}{l}\text { The number of primary users with } \\
\text { RF energy }\end{array}$ \\
\hline$e_{e}$ & $3.32 \times 10^{-7} \mathrm{~J} / \mathrm{bit}$ & $\begin{array}{l}\text { The energy consumed by the device } \\
\text { electronics per bit }\end{array}$ \\
\hline $\mathfrak{F}_{n}$ & $32 \mathrm{~Kb}$ & The bit number of a packet \\
\hline $\mathscr{L}$ & $10^{-6}$ & The bit period \\
\hline$\Gamma$ & 0.8 & A factor of energy harvesting efficiency \\
\hline$a$ & 1.1 & The path-loss exponent \\
\hline$p l_{t}$ & $\begin{array}{l}50,60,70,80,90, \\
\text { and } 100 \mathrm{~mW}\end{array}$ & $\begin{array}{l}\text { The available power levels for } \\
\text { secondary users }\end{array}$ \\
\hline$p_{t}$ & $100 \mathrm{~mW}$ & The power level for the primary users \\
\hline$G_{t}, G_{r}$ & 1,1 & $\begin{array}{l}\text { The number of more resource units } \\
\text { for the non-completed task }\end{array}$ \\
\hline W & $10 \mathrm{Mbps}$ & A set of each task's thresholds \\
\hline$\xi, q$ & $1,0.9$ & $\begin{array}{l}\text { Cost parameters for energy } \\
\text { consumption }\end{array}$ \\
\hline$\mu$ & 0.8 & $\begin{array}{l}\text { A control factor to control the weight } \\
\text { for each timed strategies }\end{array}$ \\
\hline $\mathscr{M}^{R}$ & $2 \mathrm{Mb}$ & The maximum size of $\mathbb{Q}$ \\
\hline $\mathscr{M}^{\mathbb{E}}$ & $20 \mathrm{~J}$ & The maximum capacity of $\mathbb{E}$ \\
\hline
\end{tabular}

- There are four different service applications; spectrum requirements are 128, 256, 384, and $512 \mathrm{Kbps}$. They are randomly generated for each network devices.

- The process for new service requests is Poisson with rate $\lambda$ (calls/s), and the range of offered load was varied from 0 to 3.0; the durations of calls are exponentially distributed.

- Initially, all secondary users have the same amount of energy (10 J).

- PUs occupy their allocated spectrum only $30 \%$ on average.

- System performance measures obtained on the basis of 100 simulation runs are plotted as functions of the service generation rate.

- For simplicity, we assume the absence of physical obstacles in the experiments.

Performance measures obtained through simulation are normalized system throughput, energy depletion probability of secondary users and social fairness. As mentioned earlier, we compare the performance of the proposed scheme with the existing schemes; the ONCCP scheme [16] and the OCAEH scheme [8].

Figure 3 shows the performance comparison of each scheme in terms of the normalized system throughput. It is estimated as the total data transmission in the CRN-RF system. From the point of view of network 


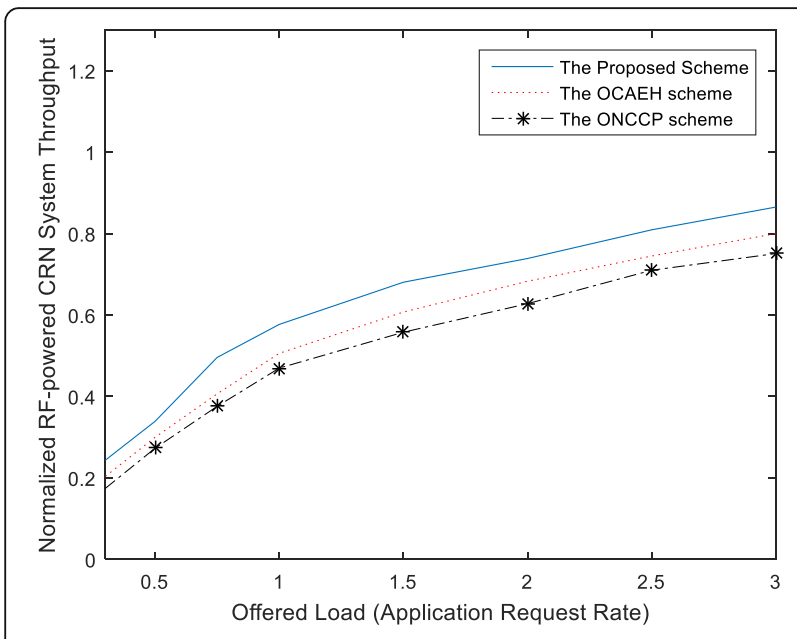

Fig. 3 Normalized CRN-RF system throughput

devices, it is an important performance metric. We observe that all the schemes produce similar performance trends; the throughput increases as the service request rate increases. In the proposed scheme, the channel look-up vector $(\mathbb{V})$ is maintained based on the timeoriented fusion rule. Therefore, unlicensed devices in our scheme can select adaptively the available channels. Due to this reason, we can get a better performance than the ONCCP and OCAEH schemes, which were onesided protocols and cannot adaptively respond the current CRN-RF system conditions.

In Fig. 4, we plot the energy depletion probability. It means a power outage ratio of unlicensed network devices. In general, as the service request rate increases, the energy depletion probability also increases. This is intuitively correct. In our game-based approach, each

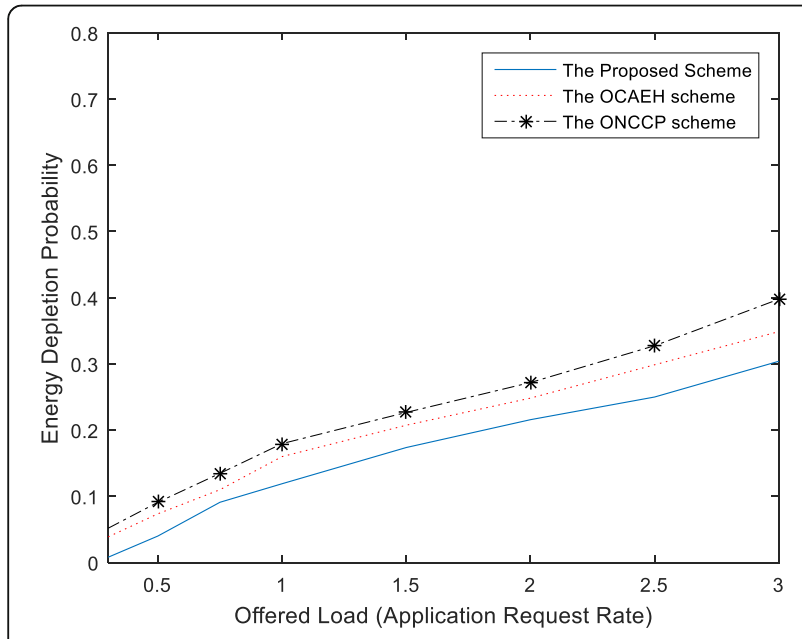

Fig. 4 Energy depletion probability network devices dynamically interact with the CRN-RF system in a distributed fashion and select their strategy based on the current energy status. Therefore, we can reduce the energy depletion probability while balancing an appropriate trade-off in harvesting energy and transferring data. Figure 4 indicates that the energy depletion probability of our proposed scheme is lower than the ONCCP and OCAEH schemes.

Figure 5 shows the different ratio of queue length in unlicensed network devices, i.e., SUs. During the CRN-RF system operation, the maximum and minimum $\mathfrak{I}$ values of SUs can be dynamically estimated. In this simulation, the different level between the maximum and minimum $\mathfrak{I}$ values in the CRN-RF system is defined as the different ratio of queue length and considered as one performance criterion. From the viewpoint of social fairness, the smaller different ratio represents that the CRN-RF system can keep the relative social fairness among network devices at the data transmission procedure. The main novelty of the proposed scheme is the sequential bargaining process to yield the maximum social benefit. Based on the individual $\mathfrak{I}$ value, the bargaining power of game players is adaptively adjusted. Therefore, our proposed scheme can effectively coordinate the data collisions and maintains a lower different ratio of queue length than other existing schemes.

The simulation results shown in Figs. 3, 4, and 5 demonstrate the performance comparison of the proposed scheme and other existing schemes $[8,16]$ and verify that the proposed game-based scheme can strike the appropriate performance balance between data transmission and RF energy harvesting. The ONCCP and OCAEH schemes cannot offer such an attractive performance balance.

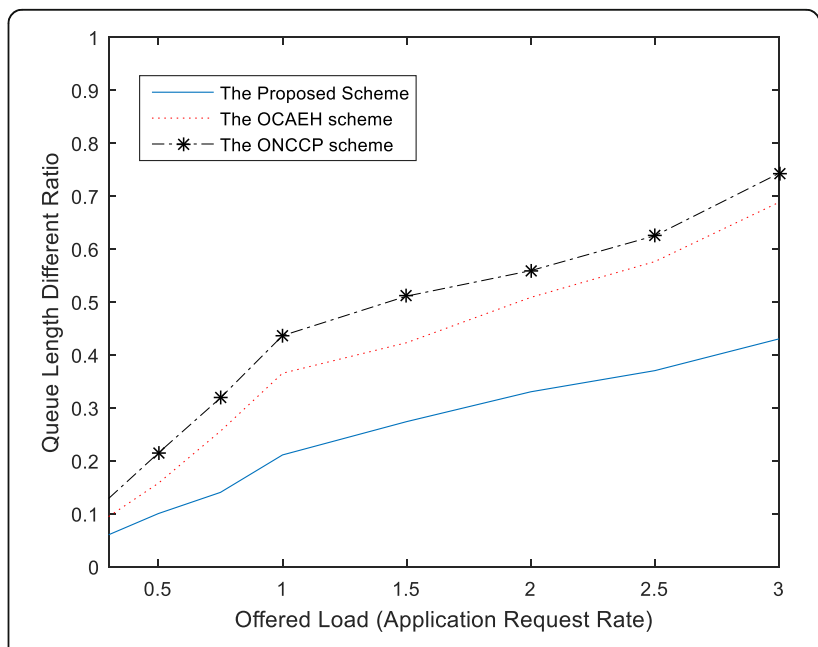

Fig. 5 Different ratio of queue length 


\section{Conclusions}

Spectrum efficiency and energy efficiency are two critical issues in designing wireless networks. As energy harvesting becomes technologically viable, RF energy harvesting has emerged as a promising technique to supply energy to wireless network devices. On the other hand, we can improve the spectrum efficiency and capacity through CR spectrum access. Currently, it has raised a demand for developing new control protocols to maximize the wireless information transferring and energy harvesting simultaneously. In this study, we design a new energyharvesting scheme for RF-CRN systems. Focusing on the trade-off between data transmission and RF energy harvesting, spectrum channels are dynamically selected and adaptively shared by unlicensed network devices. Based on the iterative game model and sequential bargaining process, we can achieve an effective RF-CRN system performance than other existing schemes. For the future research, the open issues and practical challenges are energy trading, interference management, and distributed energy beamforming in the RF-CRN system. In addition, devising a high-gain antenna for a wide range of frequency is an another important research issue.

\section{Acknowledgements}

This research was supported by the MSIP (Ministry of Science, ICT and Future Planning), Korea, under the ITRC (Information Technology Research Center) support program (IITP-2016-H8501-16-1018), supervised by the IITP (Institute for Information \& communications Technology Promotion), and was supported by Basic Science Research Program through the National Research Foundation of Korea (NRF) funded by the Ministry of Education (NRF-2015R1D1A1A01060835).

\section{Competing interests}

The author declares that he has no competing interests.

Received: 12 June 2016 Accepted: 7 December 2016

Published online: 17 January 2017

\section{References}

1. S. Park, J. Heo, B. Kim, W. Chung, H. Wang, D. Hong, Optimal mode selection for cognitive radio sensor networks with RF energy harvesting, (IEEE PIMRC'2012, 2012, Sydney), pp. 2155-2159

2. T.B. Lim, N.M. Lee, B.L. Poh, Feasibility study on ambient RF energy harvesting for wireless sensor network, (IEEE IMWS-BIO'2013, Singapore, 2013), pp. 1-3

3. I Jang, D Pyeon, S Kim, H Yoon, A survey on communication protocols for wireless sensor networks. JCSE 7(4), 231-241 (2013)

4. SMK-u-R Raazi, S Lee, A survey on key management strategies for different applications of wireless sensor networks. JCSE 4(1), 23-51 (2010)

5. E. Khansalee, Y. Zhao, E. Leelarasmee, K. Nuanyai, A dual-band rectifier for RF energy harvesting systems, (IEEE ECTI-CON'2014, Nakhon Ratchasima, 2014), pp. 1-4

6. X Lu, P Wang, D Niyato, DI Kim, H Zhu, Wireless networks with RF energy harvesting: a contemporary survey. IEEE Commun. Surv. Tutorials 17(2), 757-789 (2015)

7. $X$ Lu, $P$ Wang, D Niyato, E Hossain, Dynamic spectrum access in cognitive radio networks with RF energy harvesting. IEEE Wirel. Commun. 21(3), 102-110 (2014)

8. DT Hoang, D Niyato, P Wang, DI Kim, Opportunistic channel access and RF energy harvesting in cognitive radio networks. IEEE J. Selected Areas Commun. 32(11), 2039-2052 (2014)

9. A. Bhowmick, S.D. Roy, S. Kundu, Performance of secondary user with combined RF and non-RF based energy-harvesting in cognitive radio network (IEEE ANTS'2015, Kolkata, 2015), pp. 1-3

10. S. Kim, Game theory applications in network design, (IGI Global, Hershey, 2014)
11. D Niyato, E Hossain, MM Rashid, VK Bhargava, Wireless sensor networks with energy harvesting technologies: a game-theoretic approach to optimal energy management. IEEE Wirel. Commun. 14(4), 90-96 (2007)

12. J Zhao, W Zheng, X Wen, X Chu, H Zhang, Z Lu, Game theory based energy-aware uplink resource allocation in OFDMA femtocell networks. Int. J. Distributed Sensor Netw. 2014, 1-8 (2014)

13. H Zhang, C Jiang, NC Beaulieu, X Chu, X Wang, TQS Quek, Resource allocation for cognitive small cell networks: a cooperative bargaining game theoretic approach. IEEE Trans. Wirel. Commun. 14(6), 3481-3493 (2015)

14. W Zheng, S Tao, H Zhang, W Li, X Chu, X Wen, Distributed power optimization for spectrum-sharing femtocell networks: a fictitious game approach. J. Netw. Comput. Appl. 37, 315-322 (2014)

15. Haijun Zhang, Xiaoli Chu, Wenmin Ma, Wei Zheng and Xiangming Wen, "Resource allocation with interference mitigation in OFDMA femtocells for co-channel deployment", EURASIP J. Wireless Commun. Netw. pp.1-9, 2012

16. S. Aslam, M. Ibnkahla, Optimized node classification and channel pairing scheme for RF energy harvesting based cognitive radio sensor networks, (IEEE SSD'2015, Mahdia, 2015), pp. 1-6

17. D. Niyato, P. Wang, D.I. Kim, Admission control policy for wireless networks with RF energy transfer, (IEEE ICC'2014, Sydney, 2014), pp. 1118-1123

18. F Wei, E Jaafar MH, Energy Efficiency in Ad-hoc Wireless Networks with Two Realistic Physical Layer Models. IEEE Third International Conference on Next Generation Mobile Applications, Services and Technologies, 2009, pp. 401-406

19. B Wang, KJ Ray Liu, T Charles Clancy, Evolutionary cooperative spectrum sensing game: how to collaborate? IEEE Trans. Commun. 58(3), 890-900 (2010)

20. W Yuan, S Wen-Zhan, Cooperative resource sharing and pricing for proactive dynamic spectrum access via Nash bargaining solution. IEEE Trans. Parallel Distributed Syst. 25(11), 2804-2817 (2014)

21. D Wu, H Chen, $\mathrm{L} H \mathrm{He}, \mathrm{A}$ novel hybrid intelligence algorithm for solving combinatorial optimization problems. JCSE 8(4), 199-206 (2014)

22. J Li, J Dang, B Feng, J Wang, Analysis and improvement of the bacterial foraging optimization algorithm. JCSE 8(1), 1-10 (2014)

\section{Submit your manuscript to a SpringerOpen ${ }^{\circ}$ journal and benefit from:}

- Convenient online submission

- Rigorous peer review

- Immediate publication on acceptance

- Open access: articles freely available online

- High visibility within the field

- Retaining the copyright to your article

Submit your next manuscript at $>$ springeropen.com 